\title{
Transmission Kossel Study of Origin of Goss Texture in Grain Oriented Silicon Steel ${ }^{*}$
}

\author{
By Yukio INOKUTI,** Chizuko MAEDA,** Yo ITO** and \\ Hiroshi SHIMANAKA**
}

\begin{abstract}
Synopsis
Transmission Kossel technique has been applied in an attempt to elucidate the origin of the potential nuclei of the celebrated (110)[001] secondary grains in the high induction grain oriented silicon steel. The (110)[001] secondary recrystallization nuclei, which are inherited by the structure memory from the original hot-rolled texture, are not the recrystallized grains in the vicinity of the steel surface of the hot-rolled steel, but the polygonized (110)[001] grains elongated toward the rolling direction at about 1/10 depth from the steel surface. The polygonized (110)[001] grain consists of several matrix bands containing the transition band and normally gives the line diffusing TK patterns. There are the highly oriented (110) [001] areas with the line sharpening $T K$ patterns inside the polygonized (110)[001] grains, by which they appear to be guarded. The highly oriented (110)[001] areas are compatible with the colonies of the large primary recrystallized (110)[001] grains inherited even after the subsequent alternating operations of the cold rolling and the recrystallization annealing. It is considered that the potential nuclei of (110)[001] secondary grains will generate from the highly oriented (110)[001] areas, the preferential formation of which is indispensable for the development of the celebrated (110)[001] secondary grains during the secondary recrystallization annealing.
\end{abstract}

\section{Introduction}

The mechanism of nucleation and growth of (110) [001] secondary grains in the high induction grain oriented silicon steel is of great importance, because the perfection of (110)[001] texture in the final products gives a significant influence on the efficiency of power transformers. During past decade there has been a remarkable advance in the manufacturing process of new high induction grain oriented silicon steels; the principal innovations utilize inhibitors of $\mathrm{AlN}$ and $\mathrm{MnS},{ }^{1)} \mathrm{MnSe}$ and $\mathrm{Sb}^{2)}$ and $\mathrm{B}$ and solute $\mathrm{S}^{3 \text { ) }}$ to provide a strong inhibition to the normal grain growth. With the highly oriented products the direction of easy magnetization is closely aligned with an average deviation of only $3 \sim 4 \mathrm{deg}$ from the rolling direction of the sheet.

In contrast to these brilliant technological developments, the mechanism involved in the celebrated (110)[001] texture formation has been the subject of speculation. In the past studies the experimental techniques such as X-ray pole figure, etch-pit method, etc., have been extensively used. It has been pointed out that these conventional methods cannot obtain the detailed information about the microstructural variation. Therefore, there is no sufficient experimental evidence to allow the confident assertions about the generation of the (110)[001] secondary recrystallization nuclei.
Transmission Kossel (TK) diffraction technique, which is capable of giving the detailed informations on the microstructure of the primary and secondary grains, is very useful. In recent investigations ${ }^{4,5)}$ by use of this technique, it was discovered that the nucleation of secondary grains takes place preferentially at $30 \sim 50 \mu$ depth from the steel surface due to the structure memory from the original hot-rolled texture, and that the nuclei for secondary grains are the large primary recrystallized grains of near (110)[001] orientation formed by coalescence of several subgrains.

The object of the present work is to investigate the origin of the nucleation of (110)[001] secondary grains in the hot-rolled steel with the aid of TK diffraction technique and thereby to elucidate the mechanism of (110)[001] textural development in the high induction grain oriented silicon steel.

\section{Specimen Preparation and Experimental Procedure}

The starting material used in the present investigation was the hot-rolled sheets of about $2.7 \mathrm{~mm}$ thickness with the chemical composition of $3.25 \% \mathrm{Si}, 0.06$ $\% \mathrm{Mn}, 0.023 \%$ Se and $0.025 \% \mathrm{Sb}$. The final product made from this hot-rolled sheet has the trademark of "RG-H", which has been manufactured by twostage cold rolling process with an intermediate annealing.

Because the hot-rolled steel sheet is characterized by distinct microstructural inhomogeneities and multicomponent textures, the metallographic and textural examinations were initially done by the optical microscope and inverse pole figure method, respectively. In the latter examination the variation of texture through the thickness of the hot-rolled sheet was conducted. Successive measurements were made after the removal of appropriate layers of steel by chemical thinning, light grinding for flatness and parallelism and repolishing.

To elucidate the origin of (110)[001] texture formation, the specimens taken from 1/20 depth and 1/10 depth under the steel surface and at the center of the sheet thickness were used in TK diffraction and Shulz method. For the former TK examination thin samples of about $10 \mu$ in thickness were prepared. Representative areas of the microstructure were selected and photographically recorded. The orientations of grains in the sample were determined by using the newly developed TK apparatus. ${ }^{6}$ ) The details of the

* Presented to the 103rd ISIJ Meeting, April 1982, S545, at Tokyo Institute of Technology in Tokyo. Manuscript received September 2, 1982. (C) 1983 ISIJ

** Research Laboratories, Kawasaki Steel Corporation, Kawasaki-cho, Chiba 260. 
TK examination and analysis have been reported elsewhere. ${ }^{\text {) }}$

\section{Experimental Results}

1. Variations of Texture and Structure through the Thickness of the Hot-rolled Steel

Figure 1 shows the variation of intensities of (110) and (200) planes measured through the sheet thickness from the steel surface to the center of the hotrolled steel. The microstructure is also shown in the bottom micrograph of Fig. 1. As can be seen in Fig. 1, the intensity of (110) plane at $1 / 10$ depth from the steel surface is exceedingly strong, whereas that of (200) plane manifests the maximum value in the neighborhood of the center of the sheet thickness. A remarkable variation in the texture of the through-thickness is compatible with the past experimental results reported by Gol'dshteyn et al. ${ }^{8)}$ and Matsuo et al. ${ }^{9)}$ This fact implies that there is the origin of (110) [001] secondary recrystallization nuclei

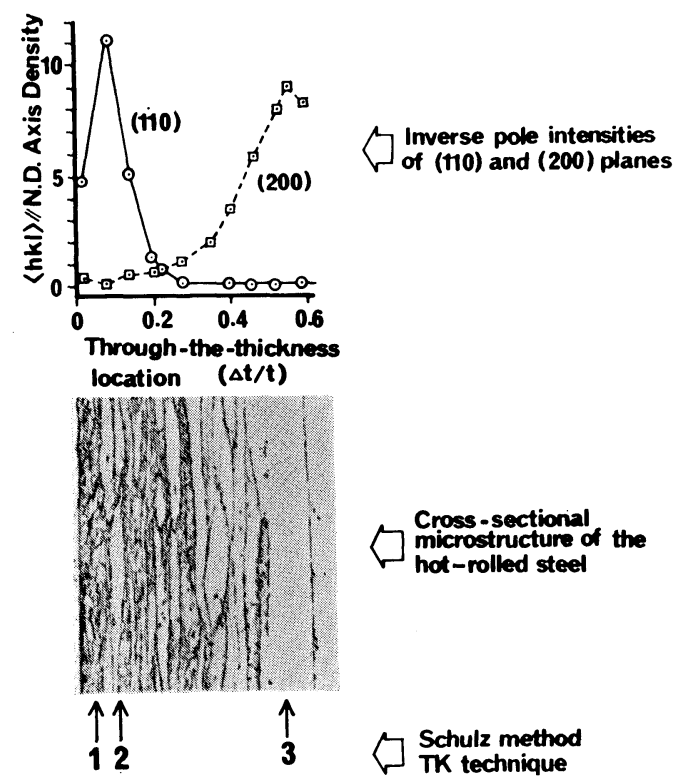

Fig. 1. The variation of intensities of (110) and (200) planes through the thickness from the surface to the center of the hot-rolled steel. The bottom micrograph shows the cross-sectional microstructure of the hot-rolled steel and the numbers of 1,2 and 3 indicate the layerwise positions of Shulz method and TK technique. in the vicinity of the surface of the hot-rolled steel rather than the center of the sheet thickness. In conjunction with the variation of texture in Fig. 1, it should be also noted that the microstructure is exceedingly inhomogeneous through the thickness of sheet; in the vicinity of the steel surface a mixed structure of the recrystallized grains and polygonized grains (deformed and unrecrystallized grains) elongated toward the rolling direction is formed, and in the central area a single structure of the more coarse polygonized grains elongated with $30 \sim 300 \mu$ width toward the rolling direction is formed.

Figure 2 shows (200) pole figures measured through the thickness of the hot-rolled steel, the corresponding positions of which are denoted with the arrow underneath the micrograph of Fig. 1. In Fig. 2 the dominant texture in the vicinity of the steel surface is $\{110\}\langle 001\rangle$, whereas that of the central area is $\{100\}\langle 011\rangle$, with strong $\langle 011\rangle$ partial fiber texture.

\section{TK Results}

\section{Structure and Texture at $1 / 10$ Depth from the Surface of the Hot-rolled Steel}

Photograph 1 shows a montage of the optical micrograph at about 1/10 depth from the surface of the hotrolled steel, where the strongest texture component of $\{110\}\langle 001\rangle$ is formed. The positions examined in detail by TK technique are denoted with the numbers in Photo. 1, in which the recrystallized and polygonized grains are labeled with the white and dark numbers, respectively.

Two distinct zones of the recrystallized grains and the extremely large polygonized grains elongated towards the rolling direction can be seen in Photo. 1. There are also two polygonized grains (1) and (2) separated straightly toward the rolling direction with a dark etched grain boundary, along which the formation of the small recrystallized grains takes place. Moreover, the central polygonized grain (1) appear to be devided into three polygonized matrix bands of (1-A), (1-B) and (1-G) with a clearly etched interface, along which the small recrystallized grains are preferentially formed. A clearly etched interface will be designated with the terminologies such as the transition band ${ }^{10)}$ or the deformation band..$^{11)}$

By the subsequent TK examination it was confirmed that the matrix bands (1-A) and (1-B) of the central polygonized grain in Photo. 1 have the crystallographic orientations within less than $10 \mathrm{deg}$ from

\section{Fig. 2.}

(200) pole figures of $1 / 20$ depth (1), 1/10 depth (2) from the surface and the center (3) of the sheet thickness of the hot-rolled steel.

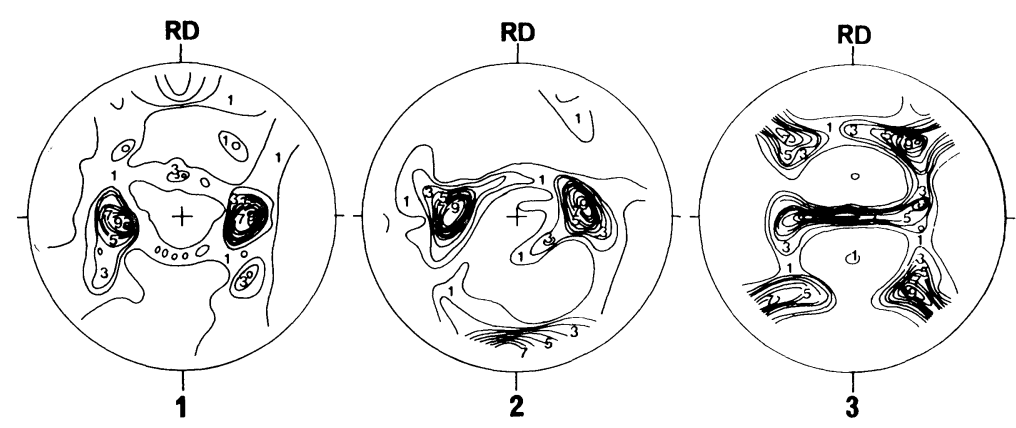


(110)[001] orientation and the matrix band (1-C) has the orientation deviated to $2 \sim 14 \mathrm{deg}$ in the crystallographic plane from (110)[001]. In contrast, the matrix bands (2-D) and (2-E) of the bottom polygonized grain (2) have the orientation of the anticlockwise rotation of $4 \sim 18 \mathrm{deg}$ about the rolling plane normal of (110)[001] orientation. Figure 3 shows the stereographic projection of the (200) poles obtained from TK patterns from the positions of the polygonized (110)[001] matrix bands (1-A), (1-B) and (1-C) of the central grain (1) in Photo. 1. The similar results of the bottom grain, which can be divided in two regions of (2-D) and (2-E) from the orientation analysis, are also given in Fig. 3. The polygonized (110)[001] matrix bands in the central grain in Photo. 1 are slightly misoriented with each other and they have the lattice curvature of $6 \sim 12$ deg within each matrix band itself. In contrast, the bottom polygonized grain in Photo. 1 has the large lattice curvature of $14 \mathrm{deg}$.

Although many of TK patterns taken from the polygonized grains gave the diffusing lines* similar to the ordinally deformed material, some TK patterns gave the sharpening lines* like the single crystal char-

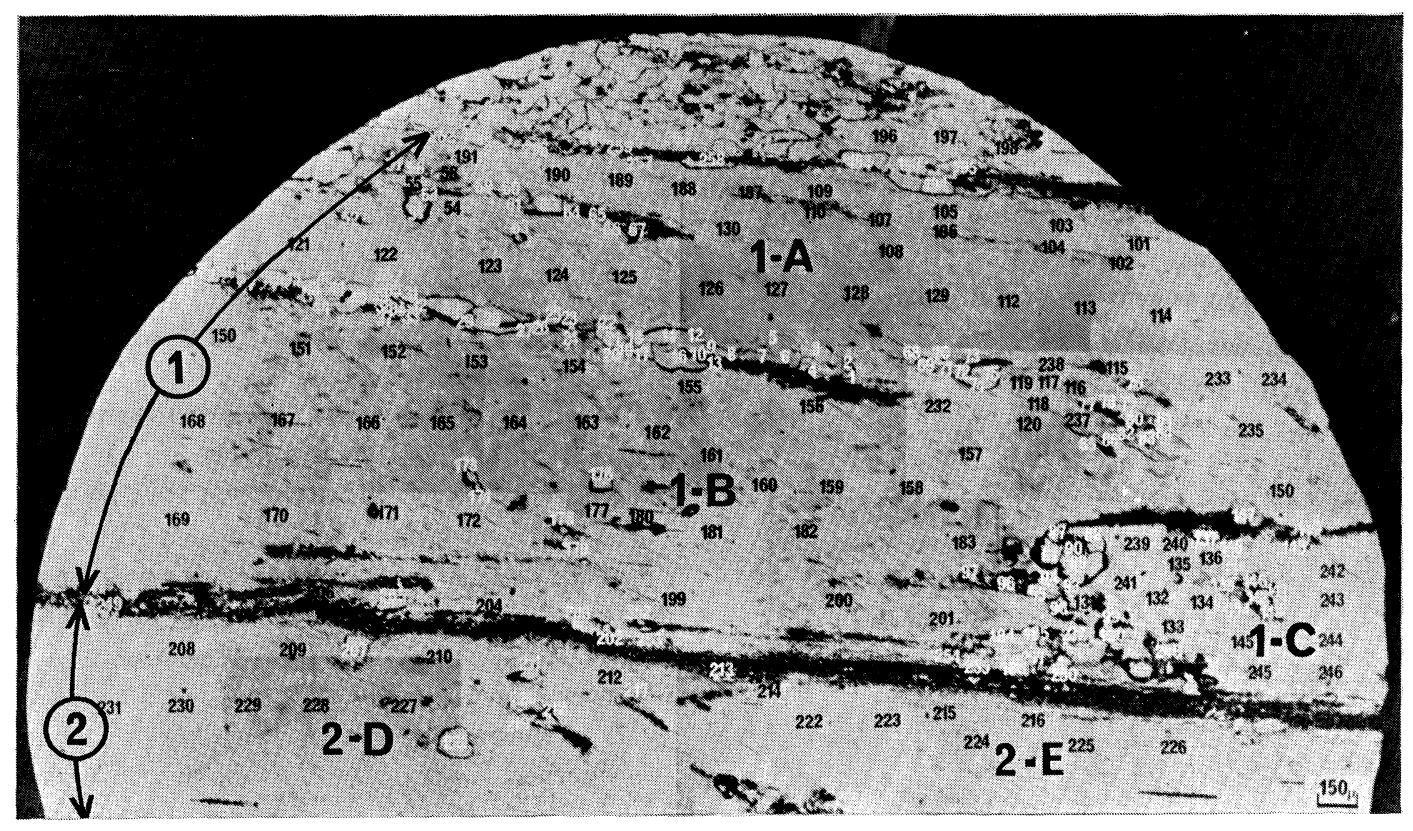

RD

Photo. 1. A montage of the optical micrograph at 1/10 depth from the steel surface of the hot-rolled steel.

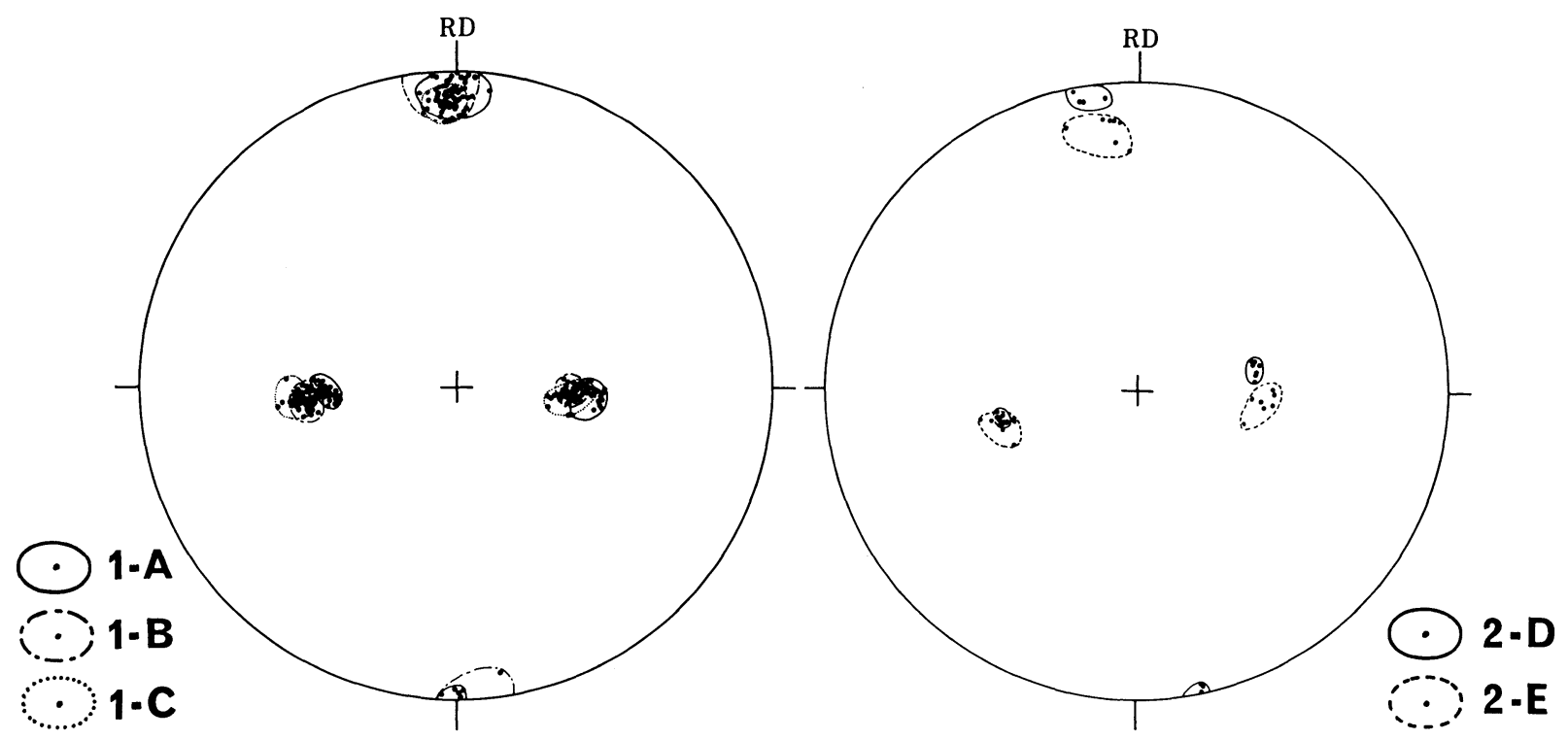

Fig. 3. The stereographic projections of (200) poles obtained from TK patterns from the positions of (A) to $(\mathrm{E})$ areas in Photo. 1 .

\footnotetext{
* The sharpening lines denote TK patterns giving sharp lines both of strong $\mathrm{FeK} \alpha_{1}$ and weak $\mathrm{FeK} \alpha_{2}$, whereas the diffusing lines denote TK patterns giving only the spread of $\mathrm{FeK} \alpha_{1}$ lines, due to the existence of strain.
} 
acteristic of new grains. The highly oriented (110) [001] areas with the line sharpening TK patterns are illustrated with the shaded marking in Photo. 2. It is worthy of note that there are four highly oriented (110)[001] areas with the line sharpening TK patterns formed at about $100 \sim 1000 \mu$ in length and about $100 \mu$ in width inside the polygonized (110) [001] matrix bands (1-A) and (1-B) of the central grain (1) in Photo. 2, but no morphological variation such as the sub-boundary separating the grain boundary in these areas can be found. However, in the bottom polygonized grain (2) of Photo. 2, no TK patterns with the sharpening line can be detected.

In order to elucidate the formation of the highly oriented (110)[001] areas, successive TK patterns were attempted to examine in more detail at the interval of about $15 \mu$ nearly perpendicular to the rolling direction in the neighborhood of the highly oriented (110)[001] area. A representative example due to the examination of successive TK patterns is shown in Photo. 3, where the white and dark numbers denote the line sharpening and diffusing TK

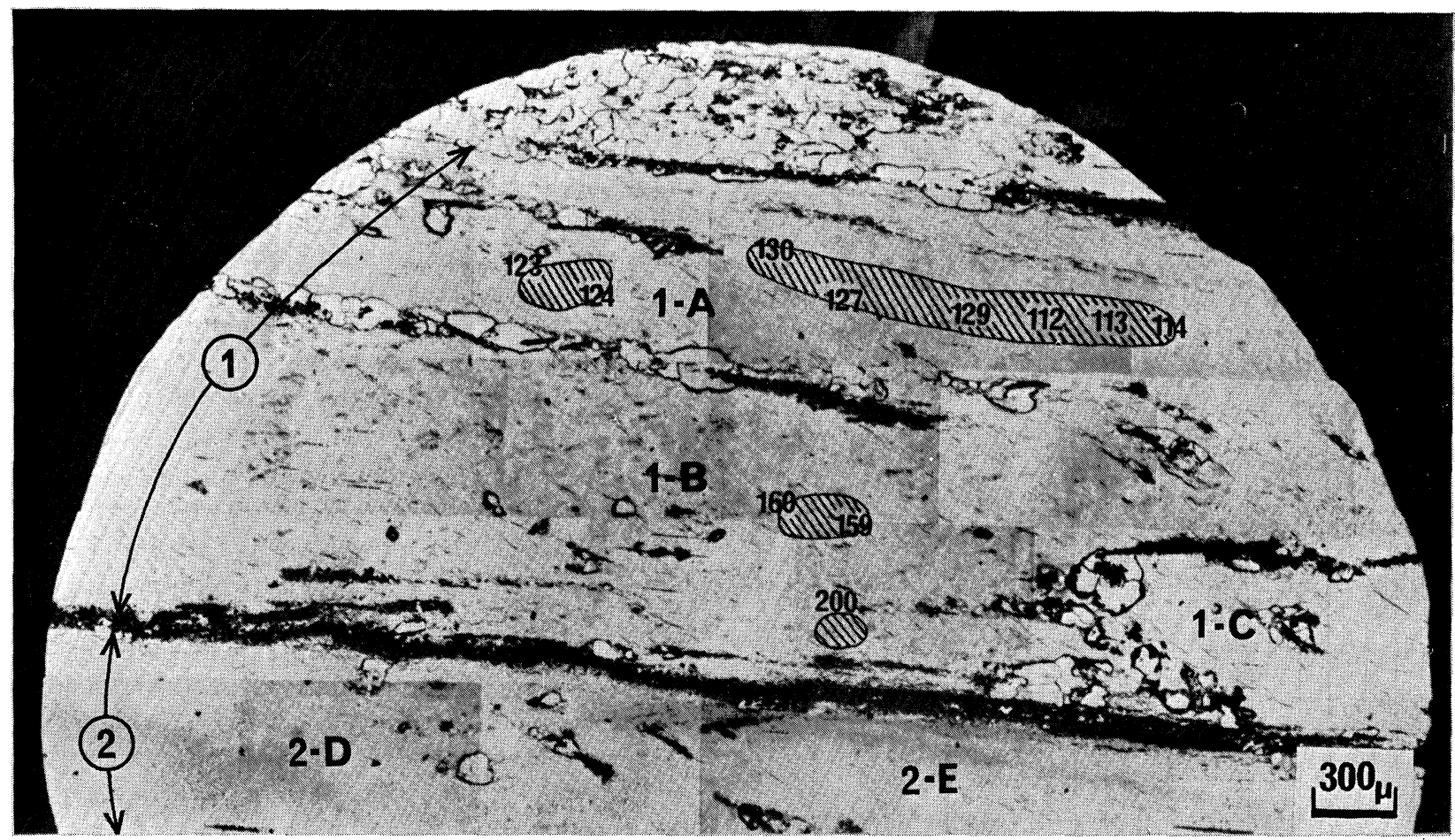

$\rightarrow$ RD

Photo. 2. The highly oriented (110) [001] areas with the line sharpening TK patterns taken from the polygonized (110) [001] matrix bands.

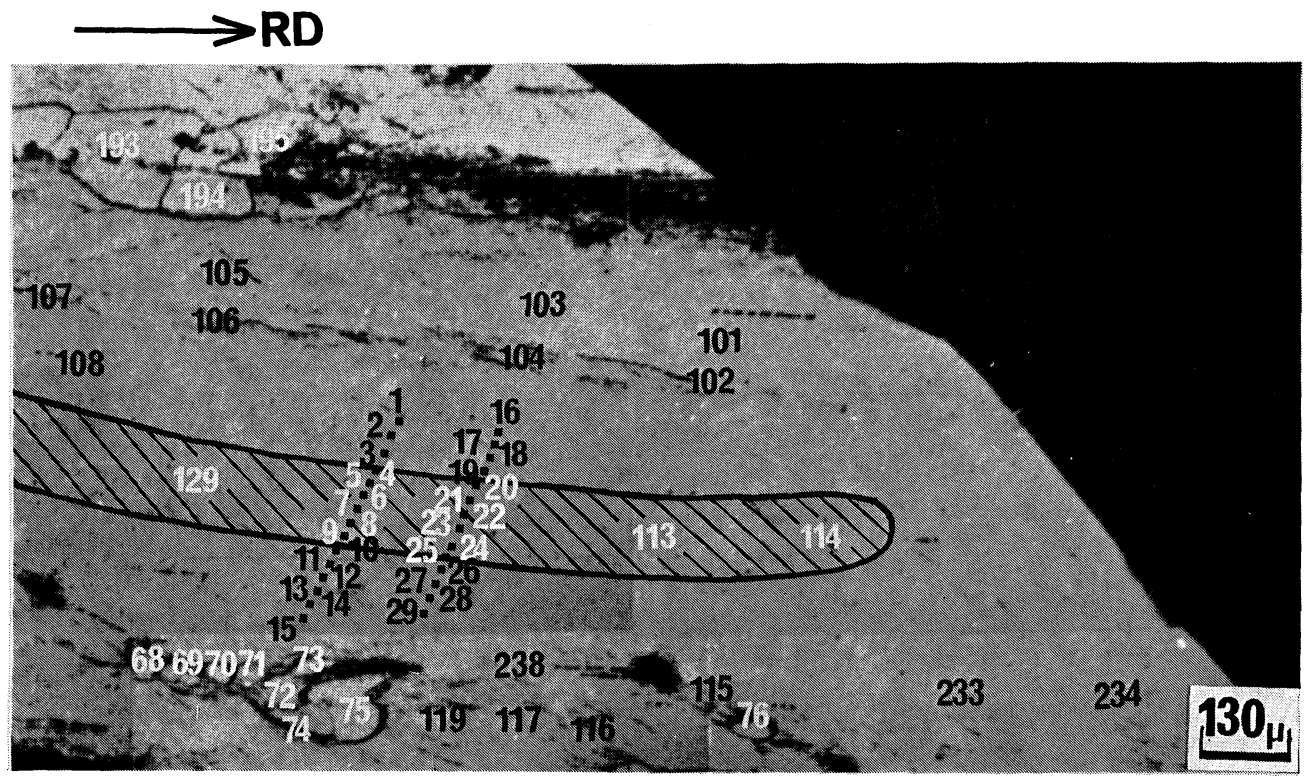

Photo. 3. The micrograph showing positions of TK patterns taken in the vicinity of the highly oriented (110) [001] area. The white and dark numbers denote the line sharpening and diffusing TK patterns, respectively. 
patterns, respectively. Also, to assess the line broadening of TK pattern, the microphotometer was used. The results of the $\mathrm{FeK} \alpha_{1}$ (110) line of TK pattern measured by the microphotometer are given in Fig. 4 . For comparison purpose similar result of new grain is shown in Fig. 4. It is evident from Fig. 4 that the broadening of (110) lines obtained from the highly oriented (110)[001] area is nearly equivalent to that of new grain. In contrast, the broadening of (110) line obtained from the polygonized (110)[001] matrix bands is approximately four times as large as that from the highly oriented (110)[001] area. Moreover, it was ascertained by TK analysis that the transition from the area of the line sharpening TK patterns to the line diffusing one changes gradually in the orientation and strain. This phenomenon is

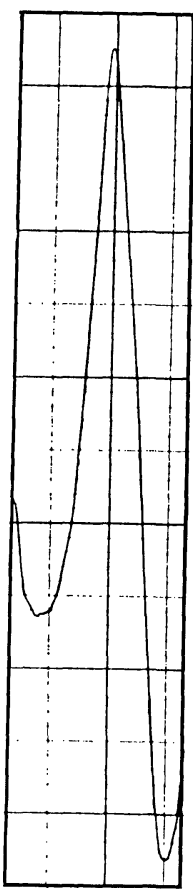

A

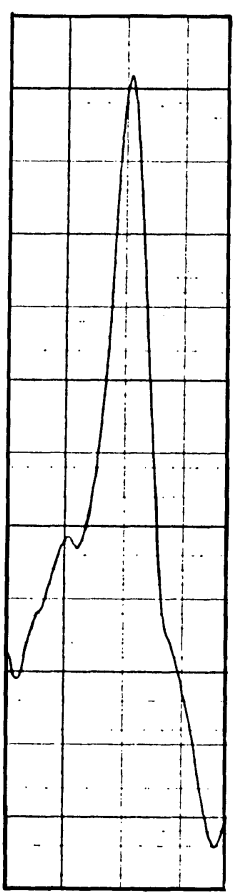

B

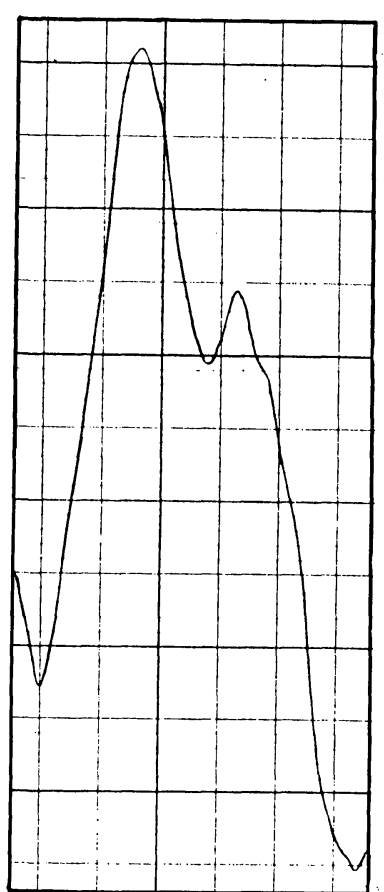

C
Fig. 4. The $\mathrm{FeK} \alpha_{1}$ (110) line of TK patterns measured by the microphotometer, where (A) to (C) represent $\mathrm{FeK} \alpha_{1}$ (110) line of the new grain, the highly oriented (110) [001] area (TK position 5 in Photo. 3 ) and the polygonized (110) [001] matrix band (TK position 1 in Photo. 3), respectively. quite different from the abrupt changes of TK patterns seen in the grain boundaries. It is important to point out that the coarse polygonized (110)[001] matrix bands elongated toward the rolling direction guard carefully the highly oriented (110)[001] areas with the line sharpening TK patterns. As will be discussed later, it is supported that these highly oriented (110)[001] areas will be the origin of (110)[001] secondary recrystallization nuclei of the hot-rolled steel.

On the other hand, in the recrystallized grains of Photo. 1, there are also two kinds of TK patterns having the diffusing and sharpening lines. Two kinds of TK patterns in the recrystallized grains will occur due to the difference for the formation of the recrystallization during and after the hot rolling process. By the extensive TK search it was found out that many TK patterns with the diffusing lines can be obtained in the recrystallized grains originated from the transition band separating the polygonized (110)[001] matrix bands, whereas those with the sharpening lines can be obtained in the recrystallized grains originated from the transition bands, grain boundaries and highly curved positions of the polygonized (110)[001] matrix bands.

The representative micrograph of the recrystallized grains originated preferentially along the transition band between the polygonized (110)[001] matrix bands (1-A) and (1-B) is shown in Photo. 4 , in which the recrystallized grains of the line sharpening TK patterns are marked with the white hyphen. The related stereographic projection of (200) poles obtained from TK patterns is also given in Fig. 5, in which the recrystallized grains with the line diffusing and line sharpening TK patterns are denoted with the marking of white and dark triangles, respectively. In Photo. 4 and Fig. 5 most of the recrystallized grains with the line diffusing TK patterns have near $\{110\}\langle 001\rangle,\{110\}\langle 112\rangle$ and $\{110\}\langle 111\rangle$ orientations, whereas those with the line sharpening TK patterns have near $\{110\}\langle 112\rangle$ and $\{110\}\langle 111\rangle$ orientations. In addition, the average grain sizes of the recrystallized grains with the line diffusing and line sharpening TK patterns are 40 and $85 \mu$, respectively. It should be emphasized that all of recrystallized $\{110\}\langle 001\rangle$ grains originated from the transition bands are the comparatively small sizes of $20 \sim 50 \mu$

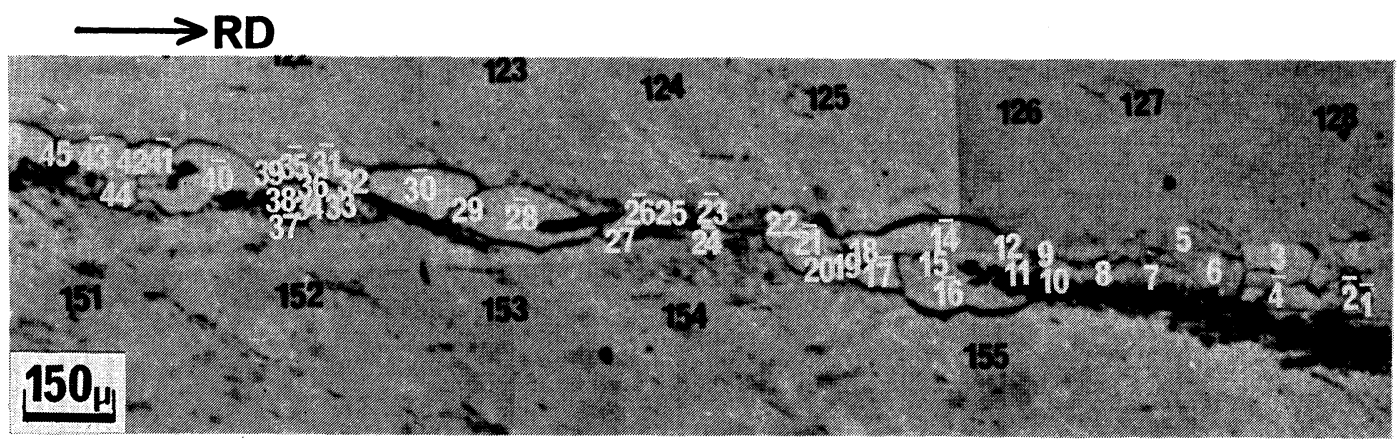

Photo. 4. The micrograph of the recrystallized grains originated from the transition band between the polygonized (110) [001] matrix bands (A) and (B). 


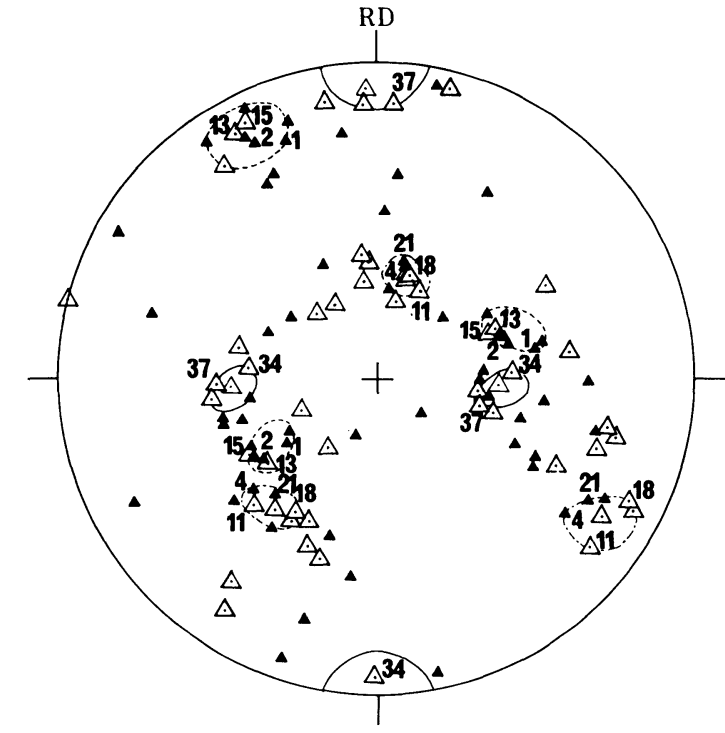

ه Recrystallized grain-line diffusing TK pattern

- Recrystallized grain-line sharpening TK pattern

Fig. 5. The stereographic projection of (200) poles obtained from TK patterns from the positions of the recrystallized grains originated along the transition band between the polygonized (110) [001] matrix bands (A) and (B) in Photo. 1.

and they are preserving a small amount of strain within each grain itself. In Fig. 6 the similar stereographic projection about the recrystallized grains originated from the grain boundaries separating two polygonized grains and two distinct zones of the recrystallized and polygonized grains in Photo. 1 is shown. It is evident from Fig. 6 that most of the recrystallized grains originated from the grain boundaries have the orientation of the clockwise rotation of $7 \sim 35 \mathrm{deg}$ about the rolling plane normal of (110) [001] orientation, and the recrystallized (110)[001] grains manifest both of the line sharpening (e.g., 248 and 256) and line diffusing TK patterns (e.g., 205) with comparatively small sizes of $20 \sim 80 \mu$.

\section{Structure and Texture at $1 / 20$ Depth from the Surface of the Hot-rolled Steel}

Photograph 5 shows a montage of the optical micrograph at about $1 / 20$ depth from the surface of the hot-rolled steel where the strong $\{110\}\langle 001\rangle$ texture forms, but the intensity of $\{110\}\langle 001\rangle$ is weaker than that of Photo. 1. Although there is a mixed structure of the recrystallized and polygonized grains in Photo. 5, the substantial volume of the polygonized grains decreases apparently in comparison with that of Photo. 1. This is in conformity with the microstructural variations through the sheet thickness in Fig. 1.

Figure 7 shows seven stereographic projections of (200) poles obtained from TK patterns from the positions of areas (A) to (G) in Photo. 5. In the connection of the microstructural inhomogenites of Photo. 5 and multicomponent textures of Fig. 7, two kinds of areas can be classified; areas (B), (C), (E) and (F) form the large volume fraction of the polygonized

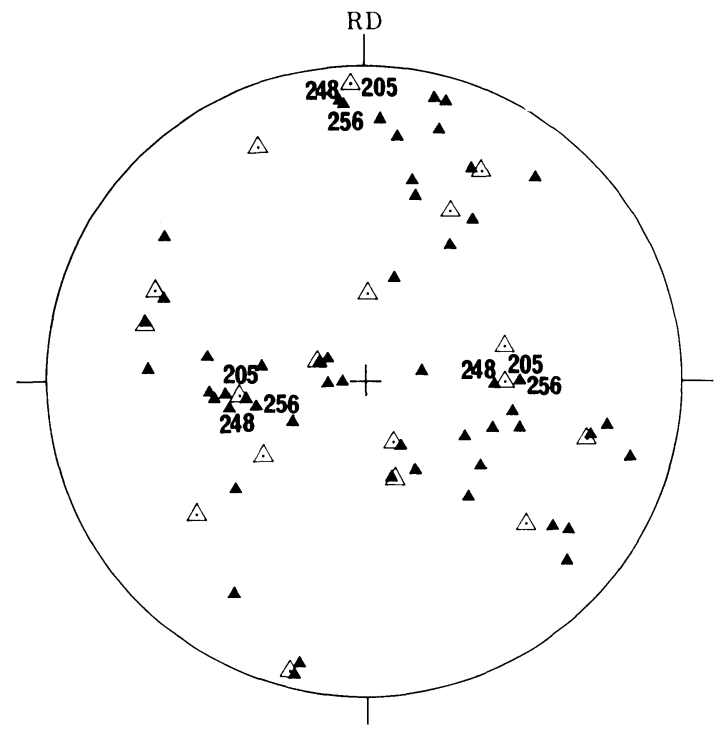

$\triangle$ Recrystallized grain-line diffusing TK pattern

- Recrystallized grain-line sharpening TK pattern

Fig. 6. The stereographic projection of (200) poles obtained from TK patterns from the positions of the recrystallized grains originated from the grain boundaries separating two polygonized grains and two distinct zones of the recrystallized and polygonized grains in Photo. 1 .

grains, and areas (A), (D) and (G) form that of the recrystallized grains. The polygonized grains of (B), (C) and (F) have the orientation with the clockwise rotation of $2 \sim 20 \mathrm{deg}$ about the rolling plane normal of (110)[001] orientation, whereas the comparatively small polygonized grain of (E) has near (110)[112] orientation. The lattice curvatures of these polygonized matrix bands of $(\mathrm{B}),(\mathrm{C}),(\mathrm{E})$ and $(\mathrm{F})$ are 21, 20, 20 and $25 \mathrm{deg}$, respectively. Also, areas of the polygonized (110)[001] grains, which have the orientations within less than $10 \mathrm{deg}$ from (110)[001], are illustrated with the shaded markings in Photo. 6. As can be seen in Photo. 6, the substantial volume of the polygonized (110)[001] grains with the shaded markings is quite large in area $(\mathrm{F})$ and small in areas $(B)$ and $(\mathrm{C})$. They are about one fifth as large as that of total polygonized grains. TK patterns with the diffusing lines characteristic of the deformed material could be only obtained in these polygonized grains, and no TK patterns with the sharpening lines, as well as those discovered in the shaded marking in Photo. 2, could be detected. In addition, in the comparison of the layerwise analysis in Photos. 1 and 5 it should be emphasized that all of the polygonized grains at $1 / 20$ depth under the surface are able to create preferentially the precise (110) plane with the moderate deviation from $\langle 001\rangle$ orientation.

With respect to a few recrystallized grains in areas (B), (C), (E) and (F) of Photo. 5 there are also two kinds of TK patterns having the diffusing and sharpening lines. Although no clear tendency in the orientation distribution of the recrystallized grains between the line diffusing and line sharpening TK patterns can 


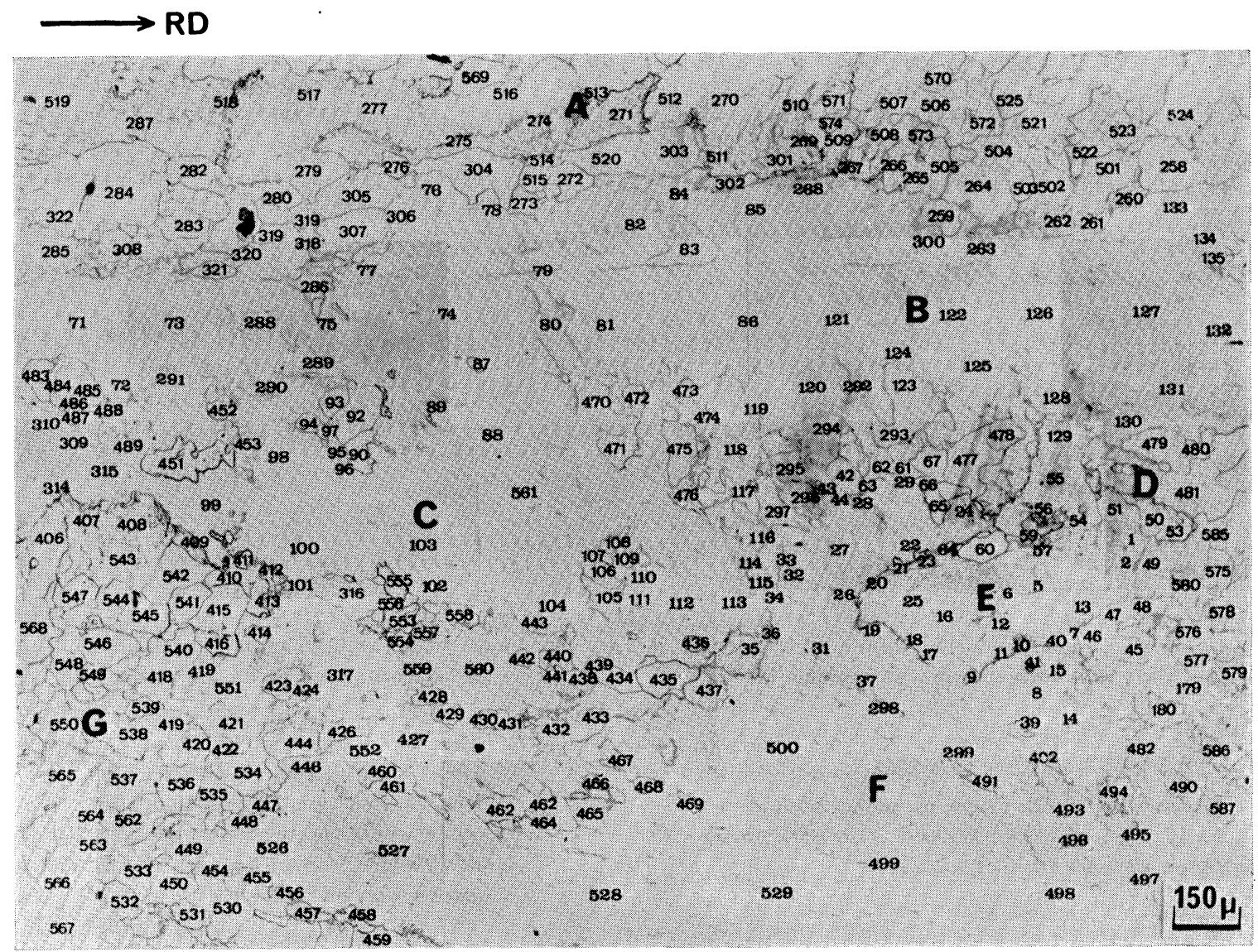

Photo. 5. A montage of the optical micrographs at $1 / 20$ depth from the steel surface of the hot-rolled steel.

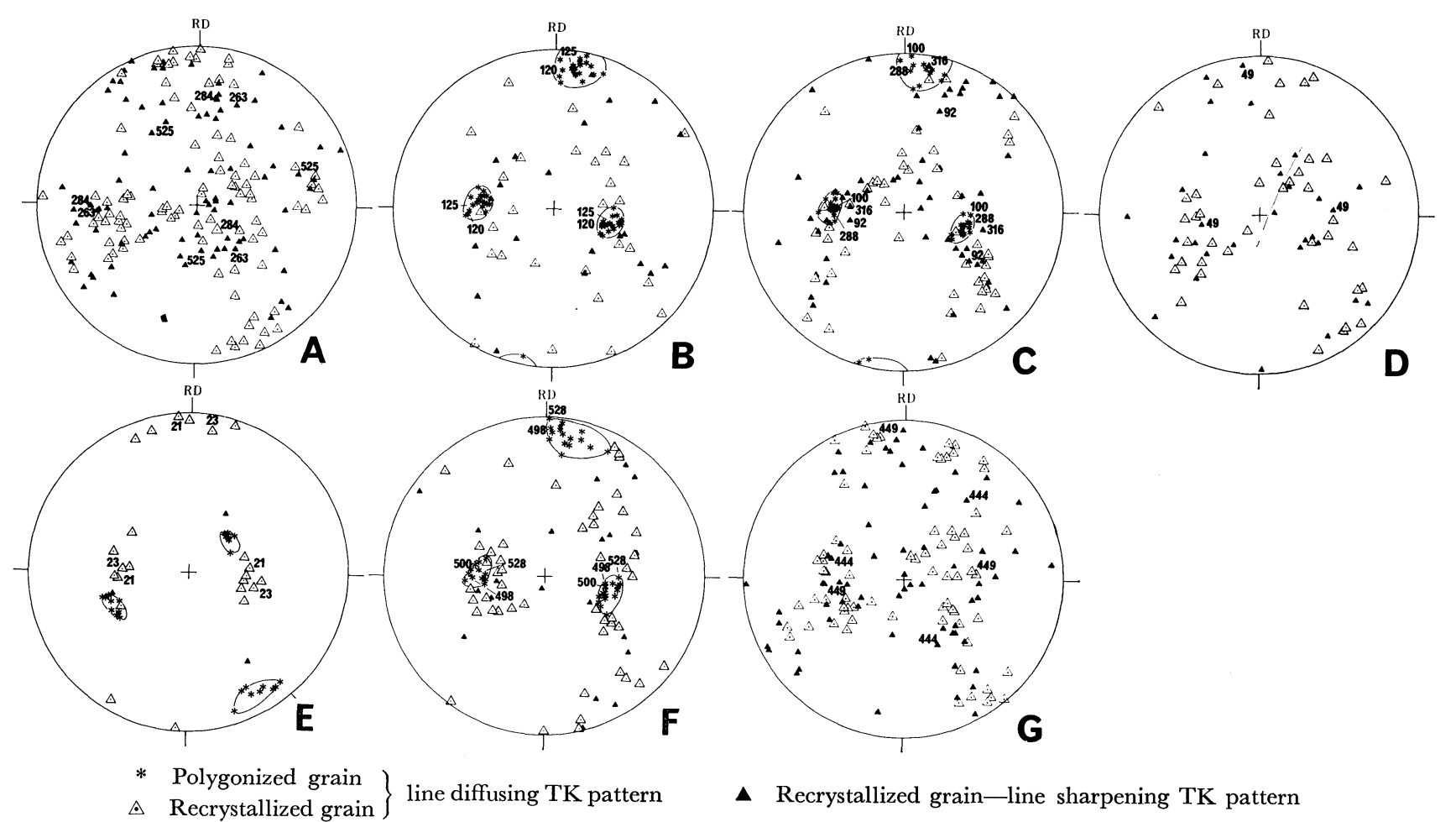

Fig. 7. The stereographic projections of (200) poles obtained from TK patterns from the positions of areas (A) to (G) in Photo. 5.

be seen, most of the recrystallized grains originated along the transition band between the polygonized grains $(\mathrm{E})$ and $(\mathrm{F})$ have near $\{110\}\langle 001\rangle$ orientation (e.g., 21 and 23) and all of them are the small size of $10 \sim 20 \mu$ with the line diffusing TK patterns.

On the other hand, in areas (A), (D) and (G) in 


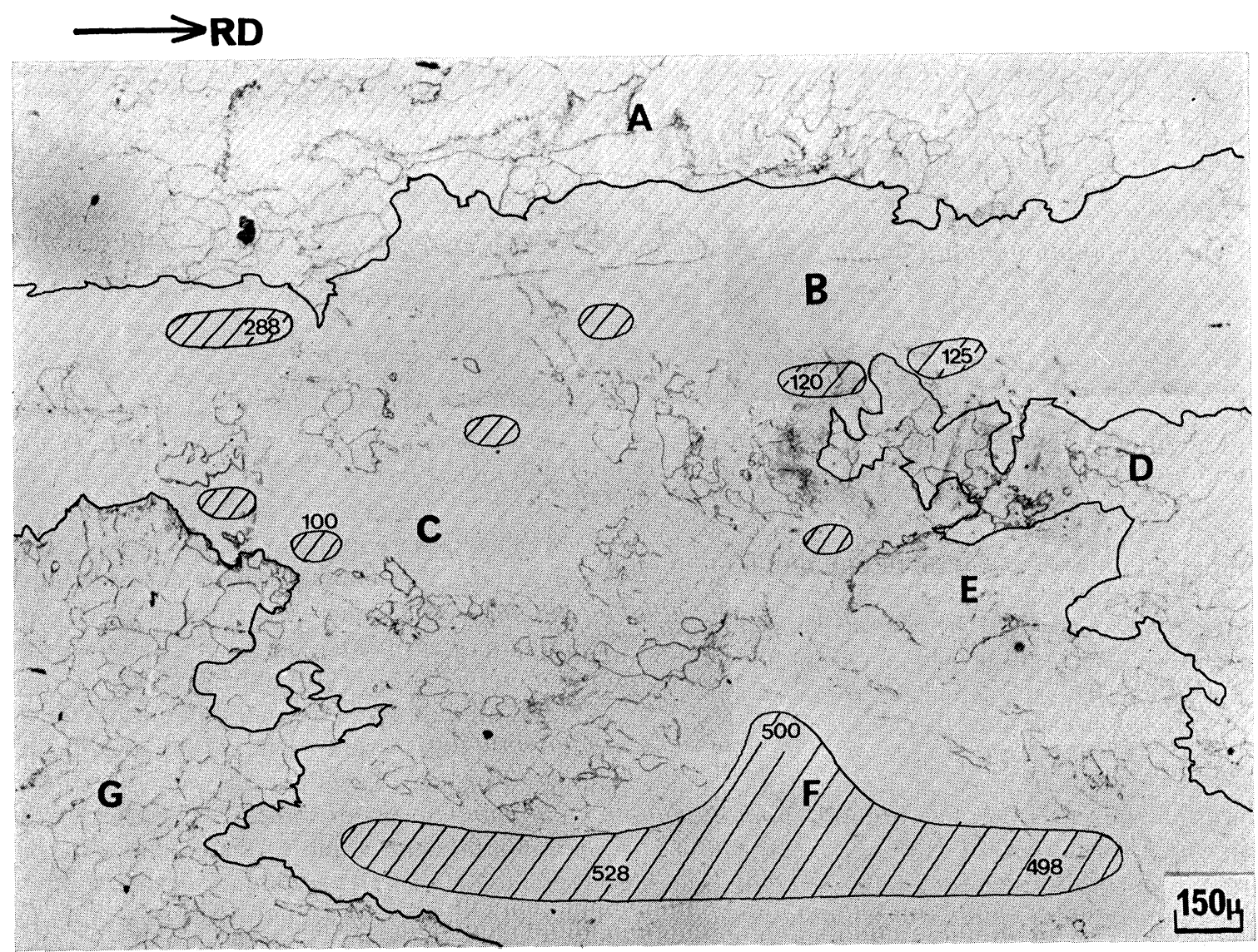

Photo. 6. The micrograph of areas having the crystallographic orientation within less than 10 deg from (110) [001] orientation among the polygonized grains in Photo. 5.

which the formation of the recrystallized grains predominates, two kinds of TK patterns having the diffusing and sharpening lines can be also obtained. The orientation distribution of the recrystallized grains with the line sharpening TK patterns is nearly random, whereas that with line diffusing TK patterns have a weak $\{110\}\langle 112\rangle$ orientation.

\section{Structure and Texture at the Gentral Area of the Hot- rolled Steel}

Photograph 7 shows a montage of the optical micrograph of the polygonized grains elongated towards the rolling direction at the central area of the hotrolled steel. Figure 8 gives the related stereographic projection of (200) poles.

In Photo. 7 only the polygonized grains elongated with about $300 \sim 800 \mu$ width to the rolling direction can be seen. Also, in Fig. 8 the polygonized grains of $\mathrm{B}, \mathrm{C}, \mathrm{E}, \mathrm{F}$ and $\mathrm{G}$ have near $\{112\}\langle 110\rangle$ orientation, whereas those of A and D have near $\{100\}\langle 001\rangle$ and $\{113\}\langle 110\rangle$ orientations, respectively. All of TK patterns taken from the polygonized grains in Photo. 7 gave the diffusing lines similar to the ordinally deformed material. The lattice curvatures of the polygonized $\{112\}\langle 110\rangle,\{100\}\langle 001\rangle$ and $\{113\}\langle 110\rangle$ grains are 5 6, 18 and $8 \mathrm{deg}$, respectively.

\section{Discussion}

In the past X-ray pole figure studies ${ }^{8)}$ it has been pointed out that the (110)[001] grains among many recrystallized grains formed in the vicinity of the steel

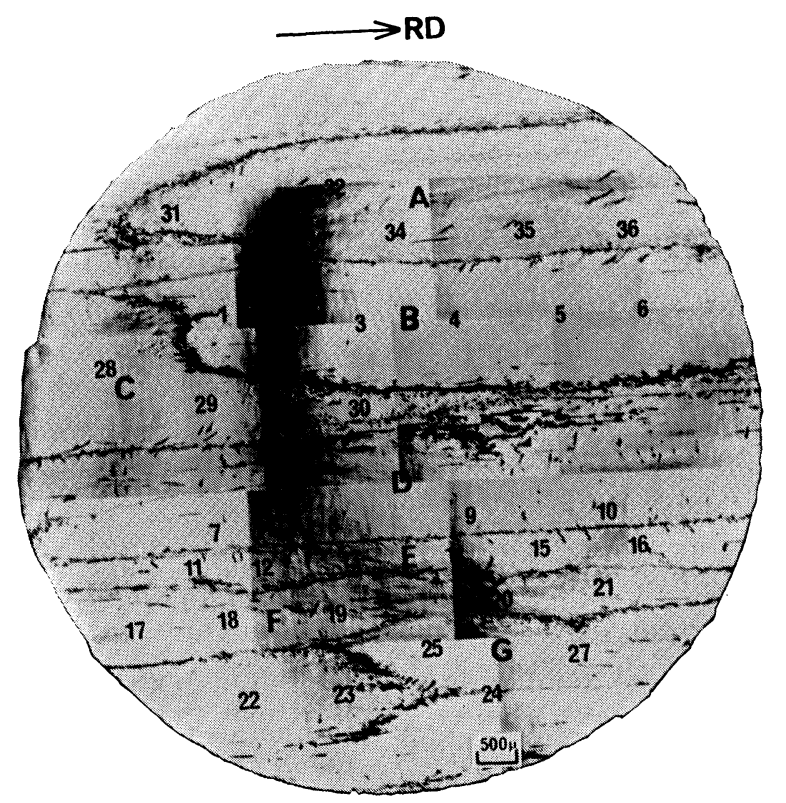

Photo. 7. A montage of the optical micrograph at the center of the thickness of the hot-rolled steel.

surface during the hot rolling will play the important role for the origin of $\{110\}\langle 001\rangle$ secondary recrystallization nuclei. Unfortunately, there has been no detailed experimental evidence to allow confident assertions concerning the origin of (110)[001] texture generation in the hot-rolled steel. Recently, in the detailed TK examination of as-decarburized and pri- 


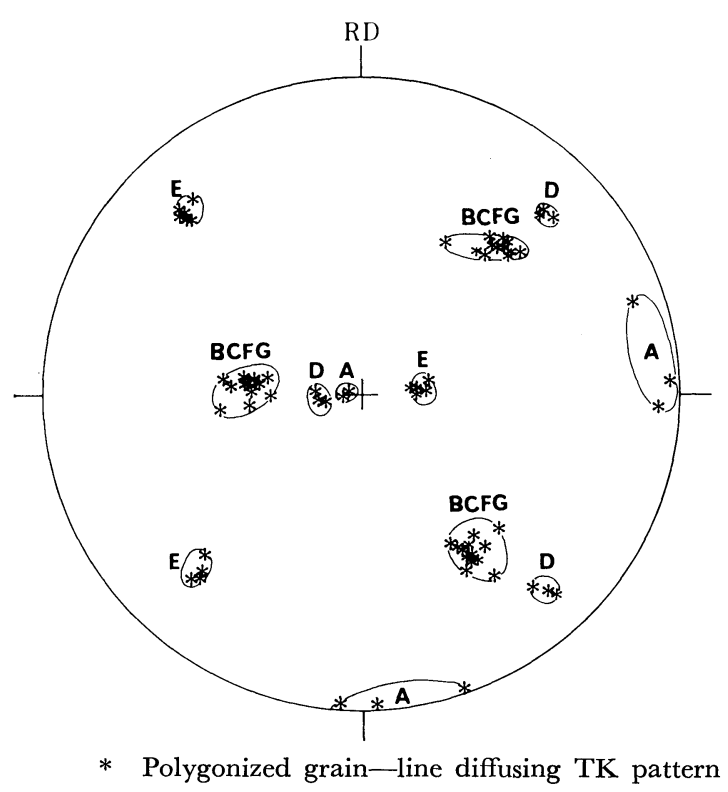

Fig. 8. The stereographic projection of (200) poles obtained from TK patterns from the positions of areas (A) to (G) in Photo. 7.

mary recrystallized steels, ${ }^{4)}$ it has been clarified that the preferential formation of the large (110)[001] secondary nuclei at the vicinity of the steel surface will be due to the structure memory* from the original hot-rolled texture.

In the present investigation it is pointed out that there is the highly oriented (110)[001] area of the line sharpening TK patterns guarded inside the polygonized (110)[001] matrix bands elongated toward the rolling direction at the $1 / 10$ depth from the steel surface. A schematic illustration of the highly oriented (110)[001] area (A) with the line sharpening TK patterns is given in Fig. 9. For comparison a few recrystallized $\{110\}\langle 001\rangle$ grains originated preferentially along the transition band or grain boundary of the polygonized (110)[001] grains and those formed among many recrystallized grains are also illustrated in areas (B) and (C) of Fig. 9, respectively.

On the basis of a hypothesis that the highly oriented (110)[001] area (A) illustrated with the shaded markings in Fig. 9 can be inherited by the structure memory from the original hot-rolled steel, ${ }^{4,5)}$ the constituent volume fraction for highly oriented (110) [001] areas is compatible with that for the colonies of large primary recrystallized (110)[001] grains within an elongated matrix zone formed even after the subsequent two-stage cold rolling with an intermediate annealing and then as-decarburized and recrystallized annealing prior to secondary recrystallization. The layerwise analysis for the highly oriented (110)[001] areas formed at 1/10 depth from the steel surface of the hot-rolled steel is in good agreement with that for (110)[001] secondary recrystallization nuclei, which

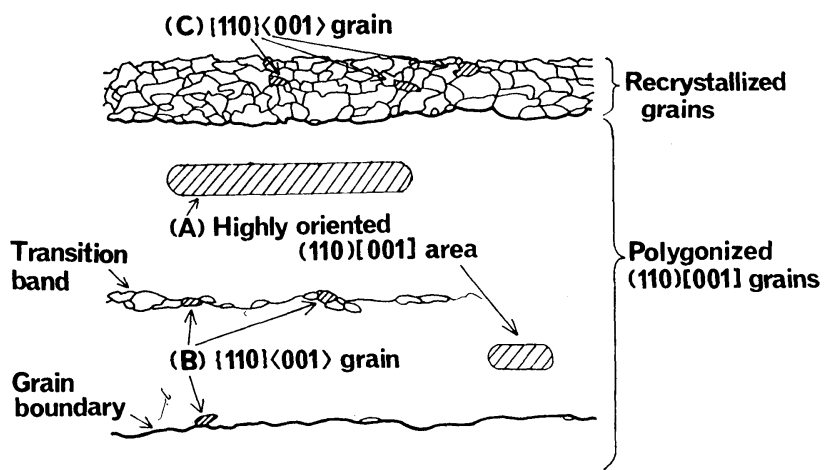

Fig. 9. The comparison of the formation of (110) [001] grains in the vicinity of the surface of the hotrolled steel.

takes place preferentially at $30 \sim 50 \mu$ depth under the steel surface. In contrast, areas (B) and (C) in Fig. 9 cannot give satisfactory explanation, due to small isolated grains of $20 \sim 80 \mu$ in size. Moreover, most of the recrystallized $\{110\}\langle 001\rangle$ grains in areas $(B)$ and (C) of Fig. 9 preserve a small amount of strain within each $\{110\}\langle 001\rangle$ grain itself. They are apt to be influenced by the adjacent grains with other orientation in the subsequent processes. Therefore, it is most plausible that the origin of the potential nuclei of the celebrated (110)[001] secondary grains in the original hot-rolled steel is illustrated with the feature like area (A) in Fig. 9. It is considered that the preferential formation of area (A) in Fig. 9 rather than that of areas (B) and (C) is indispensable for the development of the celebrated (110)[001] secondary grains during the secondary recrystallization in the high induction grain oriented silicon steel. Although the area (A) in Fig. 9 is the representative one discovered solely by TK technique, the results of which give the detailed informations on the microstructure for moderately large areas of $8 \mathrm{~mm}$ in diameter and are compatible with those of X-ray pole figures, it is generally accepted that the area (A) in Fig. 9 manifests the potential secondary recrystallization nuclei. In addition, in the layerwise analysis of the hot-rolled steel, it should be noted that the polygonized grains at somewhat inside layer (about 1/10 depth from the steel surface) can create more precise (110)[001] orientation. It will be due to avoid an unappropriate influence of the steel surface itself during the hot rolling process.

\section{Conclusions}

The results obtained are summarized in the following five items.

(1) The (110)[001] secondary recrystallization nuclei, which are inherited by the structure memory from the original hot-rolled texture, are not the small recrystallized $\{110\}\langle 001\rangle$ grains in the vicinity of the steel surface, but the polygonized (110)[001] grains

* The terminology of the structure memory has been used as the sequence of the principal $\{110\}\langle 001\rangle$ texture variation in the vicinity of the surface from the original hot-rolled steel; $\{110\}<001\rangle$ texture formation in the hot rolling $\rightarrow\{111\}\langle 11 \overline{2}\rangle$ in the first cold rolling $\rightarrow$ $\{110\}\langle 001\rangle$ in the intermediate annealing $\rightarrow\{111\}\langle 11 \overline{2}\rangle$ in the second cold rolling $\rightarrow\{110\}\langle 001\rangle$ in as-decarburized and primary recrystallized annealing. 
elongated toward the rolling direction at about $1 / 10$ depth from the steel surface.

(2) The polygonized (110)[001] grain consists of several matrix bands containing the transition band and normally gives the line diffusing TK patterns.

(3) There are the highly oriented (110)[001] areas with the line sharpening TK pattern inside the polygonized (110)[001] matrix bands, by which they appear to be guarded.

(4) Both of the constituent volume fraction and layerwise analysis of the highly oriented (110)[001] areas are compatible with those for the colonies of the large primary recrystallized (110)[001] grains within a definite elongated matrix zone, prior to the secondary recrystallization treatment.

(5) It is considered that the nucleation of the potential (110)[001] secondary grains will take place from the highly oriented (110)[001] areas, rather than the small recrystallized $\{110\}\langle 001\rangle$ grains originated from the transition band, grain boundary and highly curved positions of matrix band. The preferential formation of the highly oriented (110)[001] areas is indispensable for the development of the celebrated (110)[001] secondary grains during the secondary recrystallization annealing in the high induction grain oriented silicon steel.

\section{REFERENCES}

1) For example, S. Taguchi and A. Sakakura: J. Appl. Phys., 40 (1969), 1539.

2) I. Goto, I. Matoba, T. Imanaka, T. Gotoh and T. Kan: Proc. Soft. Magnetic Materials, 2 (1975), 262.

3) H. E. Grenoble and H. C. Fiedler: IEEE Trans. Mag., MAG-13 (1977), 1427.

4) Y. Inokuti, Y. Shimizu, C. Maeda and H. Shimanaka: Proceedings of 1st Ris $\phi$ International Symposium on Recrystallization, (1980), Ris $\phi$ National Laboratory, Denmark, 71.

5) Y. Inokuti, G. Maeda, Y. Ito and H. Shimanaka: Proceedings, The Sixth International Conference on Textures of Materials, II, ISIJ, Tokyo, (1982), 948.

6) Y. Inokuti, Y. Shimizu and H. Shimanaka: Kawasaki Steel Giho, 12 (1980), 89.

7) Y. Iwasaki, Y. Inokuti, H. Shimanaka and K. Fujimoto: Bull. Japan Inst. Metals, 18 (1979), 632.

8) V. YA. Gol'dshteyn, S. V. Pashchenko and T. A. Petrova: Phys. Met. Metall., 46 (1979), 109.

9) M. Matsuo, T. Sakai, M. Tanino, T. Shindo and S. Hayami: Proceedings, The Sixth International Conference on Textures of Materials, II, ISIJ, Tokyo, (1982), 918.

10) H. Hu: Recovery and Recrystallization of Metals, ed. by L. Himmel, Gordon \& Breach, New York, (1963), 311.

11) Y. Inokuti and R. D. Doherty: Acta Met., 26 (1978), 61. 